\title{
Discovery of an inhibitor of succinyl-CoA synthetase
}

\author{
$\mathrm{J}^{\text {Huang }}{ }^{1}$, M Fraser ${ }^{1}$ \\ ${ }^{1}$ University of Calgary, Canada \\ huang1@ucalgary.ca
}

Tartryl-CoA was discovered in the crystal structure of human GTP-specific succinyl-CoA synthetase (SCS). SCS catalyzes the only substrate-level phosphorylation of the citric acid cycle. It catalyzes the reversible reaction: succinyl-CoA $+\mathrm{NDP}+\mathrm{Pi} \rightleftharpoons$ succinate $+\mathrm{CoA}+\mathrm{NTP}$ in the presence of magnesium ions. Humans have two different SCSs, ATP-specific SCS and GTP-specific SCS. The crystallization experiment included GTP-specific SCS, ADP, CoA, and magnesium ions in the protein solution, while polyethylene glycol 3350 and ammonium tartrate were in the well solution. During crystallization, tartryl-CoA was synthesized from tartrate and CoA, and, instead of being released from the enzyme, tartryl-CoA remained as a bound ligand. The CoA portion binds as expected in the CoA-binding site, but the tartryl portion binds in the phosphate-binding site, close to the catalytic histidine residue. Although succinyl-CoA is structurally similar to tartryl-CoA, succinyl-CoA would not bind to SCS in the same way as tartryl-CoA. The two extra hydroxyl groups of tartrate contribute to the binding of tartrylCoA. Tartryl-CoA acts as an inhibitor, inhibiting SCS after a single turnover.

Acta Cryst. (2020). A76, a171 